\title{
Finite beta-expansions with negative bases
}

\author{
Zuzana Krčmáriková ${ }^{1, *}$ Wolfgang Steiner ${ }^{2, \dagger}$ Tomáš Vávra ${ }^{1, *}$ \\ ${ }^{1}$ Department of Mathematics FNSPE \\ Czech Technical University in Prague \\ Trojanova 13, 12000 Praha 2, Czech Republic \\ zuzka.krcmarikova@gmail.com, vavrato@gmail.com \\ ${ }^{2}$ IRIF, CNRS UMR 8243, Université Paris Diderot - Paris 7 \\ Case 7014, 75205 Paris Cedex 13, France \\ steiner@irif.fr
}

\begin{abstract}
The finiteness property is an important arithmetical property of beta-expansions. We exhibit classes of Pisot numbers $\beta$ having the negative finiteness property, that is the set of finite $(-\beta)$-expansions is equal to $\mathbb{Z}\left[\beta^{-1}\right]$. For a class of numbers including the Tribonacci number, we compute the maximal length of the fractional parts arising in the addition and subtraction of $(-\beta)$-integers. We also give conditions excluding the negative finiteness property.
\end{abstract}

\section{Introduction}

Digital expansions in real bases $\beta>1$ were introduced by Rényi [23]. Of particular interest are bases $\beta$ satisfying the finiteness property, or Property $(\mathrm{F})$, which means that each element of $\mathbb{Z}\left[\beta^{-1}\right] \cap[0, \infty)$ has a finite (greedy) $\beta$-expansion. We know from Frougny and Solomyak 13 that each base with Property $(\mathrm{F})$ is a Pisot number, but the converse is not true. Partial characterizations are due to [13, 16, 1]. In [2], Akiyama et al. exhibited an intimate connection to shift radix systems (SRS), following ideas of Hollander [16]. For results on shift radix systems (with the finiteness property), we refer to the survey [18].

\footnotetext{
*Supported by the Czech Science Foundation, grant No. 13-03538S, and by the Grant Agency of the Czech Technical University in Prague, grant No. SGS14/205/OHK4/3T/14

†Supported by the ANR-FWF project "Fractals and Numeration" (ANR-12-IS01-0002, FWF I1136) and the ANR project "Dyna3S" (ANR-13-BS02-0003)
} 
Numeration systems with negative base $-\beta<-1$, or $(-\beta)$-expansions, received considerable attention since the paper [17] of Ito and Sadahiro in 2009. They are given by the $(-\beta)$-transformation

$$
T_{-\beta}:\left[\ell_{\beta}, \ell_{\beta}+1\right) \rightarrow\left[\ell_{\beta}, \ell_{\beta}+1\right), \quad x \mapsto-\beta x-\left\lfloor-\beta x-\ell_{\beta}\right\rfloor, \quad \text { with } \ell_{\beta}=\frac{-\beta}{\beta+1}
$$

see Section 2 for details. Certain arithmetic aspects seem to be analogous to those for positive base systems [12, 20], others are different, e.g., both negative and positive numbers have $(-\beta)$-expansions; for $\beta<\frac{1+\sqrt{5}}{2}$, the only number with finite $(-\beta)$-expansion is 0 . We say that $\beta>1$ has the negative finiteness property, or Property $(-\mathrm{F})$, if each element of $\mathbb{Z}\left[\beta^{-1}\right]$ has a finite $(-\beta)$-expansion. By Dammak and Hbaib [10], we know that $\beta$ must be a Pisot number, as in the positive case. It was shown in [20] that the Pisot roots of $x^{2}-m x+n$, with positive integers $m, n, m \geq n+2$, satisfy the Property $(-\mathrm{F})$. This gives a complete characterization for quadratic numbers, as $\beta$ does not possess Property $(-\mathrm{F})$ if $\beta$ has a negative Galois conjugate, by [20].

First, we give other simple criteria when $\beta$ does not satisfy Property $(-\mathrm{F})$. Surprisingly, this happens when $\ell_{\beta}$ has a finite $(-\beta)$-expansion, which is somewhat opposite to the positive case, where Property (F) implies that $\beta$ is a simple Parry number.

Theorem 1. If $T_{-\beta}^{k}\left(\ell_{\beta}\right)=0$ for some $k \geq 1$, or if $\beta$ is the root of a polynomial $p(x) \in \mathbb{Z}[x]$ with $|p(-1)|=1$, then $\beta$ does not possess Property $(-F)$.

The main tool we use is a generalization of shift radix systems. We show that the $(-\beta)$-transformation is conjugated to a certain $\alpha$-SRS. Then we study properties of this dynamical system. We obtain a complete characterization for cubic Pisot units.

Theorem 2. Let $\beta>1$ be a cubic Pisot unit with minimal polynomial $x^{3}-a x^{2}+b x-c$. Then $\beta$ has Property $(-F)$ if and only if $c=1$ and $-1 \leq b<a,|a|+|b| \geq 2$.

Considering Pisot numbers of arbitrary degree, we have the following results.

Theorem 3. Let $\beta>1$ be a root of $x^{d}-m x^{d-1}-\cdots-m x-m$ for some positive integers $d, m$. Then $\beta$ has Property $(-F)$ if and only if $d \in\{1,3,5\}$.

Theorem 4. Let $\beta>1$ be a root of $x^{d}-a_{1} x^{d-1}+a_{2} x^{d-2}+\cdots+(-1)^{d} a_{d} \in \mathbb{Z}[x]$ with $a_{i} \geq 0$ for $i=1, \ldots, d$, and $a_{1} \geq 2+\sum_{i=2}^{d} a_{i}$. Then $\beta$ has Property $(-F)$.

These theorems are proved in Section 3. In Section 4, we give a precise bound on the number of fractional digits arising from addition and subtraction of $(-\beta)$-integers in case $\beta>1$ is a root of $x^{3}-m x^{2}-m x-m$ for $m \geq 1$. This is based on an extension of shift radix systems. The corresponding numbers for $\beta$-integers have not been calculated yet, although they can be determined in a similar way. 


\section{$2(-\beta)$-expansions}

For $\beta>1$, any $x \in\left[\ell_{\beta}, \ell_{\beta}+1\right)$ has an expansion of the form

$$
x=\sum_{i=1}^{\infty} \frac{x_{i}}{(-\beta)^{i}} \quad \text { with } \quad x_{i}=\left\lfloor-\beta T_{-\beta}^{i-1}(x)-\ell_{\beta}\right\rfloor \text { for all } i \geq 1 .
$$

This gives the infinite word $d_{-\beta}(x)=x_{1} x_{2} x_{3} \ldots \in \mathcal{A}^{\mathbb{N}}$ with $\mathcal{A}=\{0,1, \ldots,\lfloor\beta\rfloor\}$. Since the base is negative, we can represent any $x \in \mathbb{R}$ without the need of a minus sign. Indeed, let $k \in \mathbb{N}$ be minimal such that $\frac{x}{(-\beta)^{k}} \in\left(\ell_{\beta}, \ell_{\beta}+1\right)$ and $d_{-\beta}\left(\frac{x}{(-\beta)^{k}}\right)=x_{1} x_{2} x_{3} \cdots$. Then the $(-\beta)$-expansion of $x$ is defined as

$$
\langle x\rangle_{-\beta}= \begin{cases}x_{1} \cdots x_{k-1} x_{k} \bullet x_{k+1} x_{k+2} \cdots & \text { if } k \geq 1 \\ 0 \bullet x_{1} x_{2} x_{3} \cdots & \text { if } k=0\end{cases}
$$

Similarly to positive base numeration systems, the set of $(-\beta)$-integers can be defined using the notion of $\langle x\rangle_{-\beta}$, by

$$
\mathbb{Z}_{-\beta}=\left\{x \in \mathbb{R}:\langle x\rangle_{-\beta}=x_{1} \cdots x_{k-1} x_{k} \bullet 0^{\omega}\right\}=\bigcup_{k \geq 0}(-\beta)^{k} T_{-\beta}^{-k}(0),
$$

where $0^{\omega}$ is the infinite repetition of zeros. The set of numbers with finite $(-\beta)$-expansion is

$$
\operatorname{Fin}(-\beta)=\left\{x \in \mathbb{R}:\langle x\rangle_{-\beta}=x_{1} \cdots x_{k-1} x_{k} \bullet x_{k+1} \cdots x_{k+n} 0^{\omega}\right\}=\bigcup_{n \geq 0} \frac{\mathbb{Z}_{-\beta}}{(-\beta)^{n}} .
$$

If $\langle x\rangle_{-\beta}=x_{1} \cdots x_{k-1} x_{k} \bullet x_{k+1} \cdots x_{k+n} 0^{\omega}$ with $x_{k+n} \neq 0$, then $\operatorname{fr}(x)=n$ denotes the length of the fractional part of $x$; if $x \in \mathbb{Z}_{-\beta}$, then $\operatorname{fr}(x)=0$.

\section{Finiteness}

In this section, we discuss the Property $(-\mathrm{F})$ for several classes of Pisot numbers $\beta$. Note that $\operatorname{Fin}(-\beta)$ is a subset of $\mathbb{Z}\left[\beta^{-1}\right]$ since $\beta$ is an algebraic integer, hence Property $(-\mathrm{F})$ means that $\operatorname{Fin}(-\beta)=\mathbb{Z}\left[\beta^{-1}\right]$, i.e., $\operatorname{Fin}(-\beta)$ is a ring. We start by showing that bases $\beta$ satisfying $d_{-\beta}\left(\ell_{\beta}\right)=d_{1} d_{2} \ldots d_{k} 0^{\omega}$, which can be considered as analogs to simple Parry numbers, do not possess Property $(-\mathrm{F})$. This was conjectured in [19] and supported by the fact that $d_{-\beta}\left(\ell_{\beta}\right)=d_{1} d_{2} \ldots d_{k} 0^{\omega}$ with $d_{1} \geq d_{j}+2$ for all $2 \leq j \leq k$ implies that $d_{-\beta}\left(\beta-1-d_{1}\right)=\left(d_{2}+1\right)\left(d_{3}+1\right) \cdots\left(d_{k}+1\right) 1^{\omega}$. However, the assumption $d_{1} \geq d_{j}+2$ is not necessary for showing that Property $(-\mathrm{F})$ does not hold.

We also prove that a base with Property $(-\mathrm{F})$ cannot be the root of a polynomial of the form $a_{0} x^{d}+a_{1} x^{d-1}+\cdots+a_{d}$ with $\left|\sum_{i=0}^{d}(-1)^{i} a_{i}\right|=1$. 
Proof of Theorem 1. If $T_{-\beta}^{k}\left(\ell_{\beta}\right)=0$, i.e., $d_{-\beta}\left(\ell_{\beta}\right)=d_{1} d_{2} \ldots d_{k} 0^{\omega}$, then we have

$$
\frac{-\beta}{\beta+1}=\frac{d_{1}}{-\beta}+\frac{d_{2}}{(-\beta)^{2}}+\cdots+\frac{d_{k}}{(-\beta)^{k}}
$$

and thus $\frac{-1}{\beta+1} \in \mathbb{Z}\left[\beta^{-1}\right]$. However, we have $\frac{-1}{\beta+1} \notin \operatorname{Fin}(-\beta)$ since $T_{-\beta}\left(\frac{-1}{\beta+1}\right)=\frac{-1}{\beta+1}$, i.e., $d_{-\beta}\left(\frac{-1}{\beta+1}\right)=1^{\omega}$. Hence $\beta$ does not possess Property $(-\mathrm{F})$.

If $p(\beta)=0$ with $|p(-1)|=1$, then write

$$
p(x-1)=x f(x)+p(-1),
$$

with $f(x) \in \mathbb{Z}[x]$. Then we have $\frac{1}{\beta+1}=|f(\beta+1)| \in \mathbb{Z}[\beta]$ and thus $\frac{-(-\beta)^{-j}}{\beta+1} \in \mathbb{Z}\left[\beta^{-1}\right]$ for some $j \geq 0$. Now, $d_{-\beta}\left(\frac{-(\beta)^{-j}}{\beta+1}\right)=0^{j} 1^{\omega}$ implies that $\beta$ does not have the Property $(-\mathrm{F})$.

The main tool we will be using in the rest of the paper are $\alpha$-shift radix systems. An $\alpha$-SRS is a dynamical system acting on $\mathbb{Z}^{d}$ in the following way. For $\alpha \in \mathbb{R}, \mathbf{r}=$ $\left(r_{0}, r_{1}, \ldots, r_{d-1}\right) \in \mathbb{R}^{d}$, and $\mathbf{z}=\left(z_{0}, z_{1}, \ldots, z_{d-1}\right) \in \mathbb{Z}^{d}$, let $\tau_{\mathbf{r}, \alpha}$ be defined as

$$
\tau_{\mathbf{r}, \alpha}\left(z_{0}, z_{1}, \ldots, z_{d-1}\right)=\left(z_{1}, \ldots, z_{d-1}, z_{d}\right),
$$

where $z_{d}$ is the unique integer satisfying

$$
0 \leq r_{0} z_{0}+r_{1} z_{1}+\cdots+r_{d-1} z_{d-1}+z_{d}+\alpha<1 .
$$

Alternatively, we can say that

$$
\tau_{\mathbf{r}, \alpha}\left(z_{0}, z_{1}, \ldots, z_{d-1}\right)=\left(z_{1}, \ldots, z_{d-1},-\lfloor\mathbf{r z}+\alpha\rfloor\right),
$$

where rz stands for the scalar product.

The usefulness of $\alpha$-SRS with $\alpha=0$ for the study of finiteness of $\beta$-expansions was first shown by Hollander in his thesis [16]. His approach was later formalized in [2] where the case $\alpha=0$ was extensively studied. The symmetric case with $\alpha=\frac{1}{2}$ was then studied in [4]. Finally, general $\alpha$-SRS were considered by Surer [24].

We say that $\tau_{\mathbf{r}, \alpha}$ has the finiteness property if for each $\mathbf{z} \in \mathbb{Z}^{d}$ there exists $k \in \mathbb{N}$ such that $\tau_{\mathbf{r}, \alpha}^{k}(\mathbf{z})=\mathbf{0}$. The finiteness property of $\tau_{\mathbf{r}, \alpha}$ is closely related to the Property $(-\mathrm{F})$, thus it is desirable to study the set

$$
\mathcal{D}_{d, \alpha}^{0}=\left\{\mathbf{r} \in \mathbb{R}^{d}: \forall \mathbf{z} \in \mathbb{Z}^{d}, \exists k, \tau_{\mathbf{r}, \alpha}^{k}(\mathbf{z})=\mathbf{0}\right\} .
$$

The following proposition shows the link between $(-\beta)$-expansions and $\alpha$-SRS.

Proposition 5. Let $\beta>1$ be an algebraic integer with minimal polynomial $x^{d}+a_{1} x^{d-1}+$ $\cdots+a_{d-1} x+a_{d}$. Set $\alpha=\frac{\beta}{\beta+1}$ and let $\left(r_{0}, r_{1}, \ldots, r_{d-2}\right) \in \mathbb{R}^{d-1}$ be such that

$$
\begin{gathered}
x^{d}+(-1) a_{1} x^{d-1}+\cdots+(-1)^{d} a_{d}=(x+\beta)\left(x^{d-1}+r_{d-2} x^{d-2}+\cdots+r_{1} x+r_{0}\right), \\
\text { i.e., } \quad r_{i}=(-1)^{d-i}\left(\frac{a_{d-i}}{\beta}+\cdots+\frac{a_{d}}{\beta^{i+1}}\right) \quad \text { for } i=0,1, \ldots, d-2 .
\end{gathered}
$$

Then $\beta$ has Property $(-F)$ if and only if $\left(r_{0}, r_{1}, \ldots, r_{d-2}\right) \in \mathcal{D}_{d-1, \alpha}^{0}$. 
Proof. Let $\mathbf{r}=\left(r_{0}, r_{1}, \ldots, r_{d-2}\right)$. First we show that for $\phi: \mathbf{z} \mapsto \mathbf{r z}-\lfloor\mathbf{r z}+\alpha\rfloor$ the following commutation diagram holds, i.e., the systems $\left(\tau_{\mathbf{r}, \alpha}, \mathbb{Z}^{d-1}\right)$ and $\left(T_{-\beta}, \mathbb{Z}[\beta] \cap\left[\ell_{\beta}, \ell_{\beta}+1\right)\right)$ are conjugated.

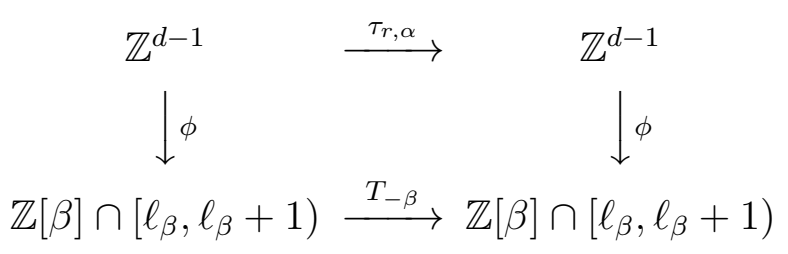

Since $r_{i}=(-1)^{d-i-1}\left(\beta^{d-i-1}+a_{1} \beta^{d-i-2}+\cdots+a_{d-i-1}\right)$ for $0 \leq i \leq d-2$, the set $\left\{r_{i}\right.$ : $0 \leq i<d\}$ with $r_{d-1}=1$ forms a basis of $\mathbb{Z}[\beta]$, hence $\phi$ is a bijection. Moreover, we have $-\beta r_{i}=r_{i-1}+c_{i}$ with $c_{i} \in \mathbb{Z}$ and $r_{-1}=0$. For $\mathbf{z}=\left(z_{0}, z_{1}, \ldots, z_{d-2}\right)$, we have $\phi(\mathbf{z})=\sum_{i=0}^{d-1} r_{i} z_{i}$ with $z_{d-1}=-\lfloor\mathbf{r z}+\alpha\rfloor$, thus

$$
T_{-\beta}(\phi(\mathbf{z}))=-\beta \phi(\mathbf{z})+n=\sum_{i=1}^{d-1} r_{i-1} z_{i}+n^{\prime}=\phi\left(z_{1}, \ldots, z_{d-2}, z_{d-1}\right)=\phi\left(\tau_{\mathbf{r}, \alpha}(\mathbf{z})\right),
$$

where $n$ and $n^{\prime}$ are integers; for the third equality, we have used that $T_{-\beta}(\phi(\mathbf{z})) \in\left[\ell_{\beta}, \ell_{\beta}+1\right)$.

Therefore, we have $\mathbf{r} \in \mathcal{D}_{d-1, \alpha}^{0}$ if and only if for each $x \in \mathbb{Z}[\beta] \cap\left[\ell_{\beta}, \ell_{\beta}+1\right)$ there exists $k \geq 0$ such that $T_{-\beta}^{k}(x)=0$. Since for each $x \in \mathbb{Z}\left[\beta^{-1}\right] \cap\left[\ell_{\beta}, \ell_{\beta}+1\right)$ we have $T_{-\beta}^{n}(x) \in \mathbb{Z}[\beta]$ for some $n \in \mathbb{N}$, Property $(-\mathrm{F})$ is equivalent to $\mathbf{r} \in \mathcal{D}_{d-1, \alpha}^{0}$.

Thus the problem of finiteness of $(-\beta)$-expansions can be interpreted as the problem of finiteness of the corresponding $\alpha$-SRS. This problem is often decidable by checking the finiteness of $\alpha$-SRS expansions of a certain subset of $\mathbb{Z}^{d}$. A set of witnesses of $\mathbf{r} \in \mathbb{R}^{d}$ is a set $\mathcal{V} \subset \mathbb{Z}^{d}$ that satisfies

1. $\pm \mathbf{e}_{i} \in \mathcal{V}$ where $\mathbf{e}_{i}$ denotes the standard basis of $\mathbb{R}^{d}$,

2. if $\mathbf{z} \in \mathcal{V}$, then $\tau_{\mathbf{r}, 0}(\mathbf{z}),-\tau_{\mathbf{r}, 0}(-\mathbf{z}) \in \mathcal{V}$.

The following proposition is due to Surer [24] and Brunotte [7].

Proposition 6. Let $\alpha \in[0,1)$ and $\mathbf{r} \in \mathbb{R}^{d}$. Then $\mathbf{r} \in \mathcal{D}_{d, \alpha}^{0}$ if and only if there exists a set of witnesses that does not contain nonzero periodic elements of $\tau_{\mathbf{r}, \alpha}$.

Sets of witnesses for several classes of $\mathbf{r} \in \mathbb{R}^{d}$ were derived in [3]. Exploiting their explicit form, several regions of finiteness can be determined; see in particular [3, Theorems 3.33.5]. An $\alpha$-SRS analogy of some of those regions was given by Brunotte [7]. Brunotte's result, however, is unsuitable for our purposes. The next proposition gives several regions of finiteness of $\alpha$-SRS.

Proposition 7. Let $\mathbf{r}=\left(r_{0}, r_{1}, \ldots, r_{d-1}\right) \in \mathbb{R}^{d}$ and $\alpha \in[0,1)$.

1. If $\sum_{i=0}^{d-1}\left|r_{i}\right| \leq \alpha$ and $\sum_{r_{i}<0} r_{i}>\alpha-1$, then $\mathbf{r} \in \mathcal{D}_{d, \alpha}^{0}$.

2. If $0 \leq r_{0} \leq r_{1} \leq \cdots \leq r_{d-1} \leq \alpha$, then $\mathbf{r} \in \mathcal{D}_{d, \alpha}^{0}$. 
3. If $\sum_{i=0}^{d-1}\left|r_{i}\right| \leq \alpha$ and $r_{i}<0$ for exactly one index $i=d-k$, then $\mathbf{r} \in \mathcal{D}_{d, \alpha}^{0}$ if and only if

$$
\sum_{1 \leq j \leq d / k} r_{d-j k}>\alpha-1
$$

Proof. 1. The set $\mathcal{V}=\{-1,0,1\}^{d}$ is closed under $\tau_{\mathbf{r}, 0}(\mathbf{z})$ and $-\tau_{\mathbf{r}, 0}(-\mathbf{z})$, hence it is a set of witnesses. For any $\mathbf{z} \in \mathcal{V}$ we have $|\mathbf{r z}| \leq \alpha$, thus $\lfloor\mathbf{r z}+\alpha\rfloor \in\{0,1\}$. Hence any periodic point of $\tau_{\mathbf{r}, \alpha}$ is in $\{0,-1\}^{d}$. For $\mathbf{z} \in\{0,-1\}^{d}$ we have $\mathbf{r z}+\alpha \leq$ $-\sum_{r_{j}<0} r_{j}+\alpha<1$. Therefore $\lfloor\mathbf{r z}+\alpha\rfloor=0$, so the only period is the trivial one.

2. In this case we take as a set of witnesses the elements of $\{-1,0,1\}^{d}$ with alternating signs, i.e., $z_{i} z_{j} \leq 0$ for any pair of indices $i<j$ such that $z_{k}=0$ for each $i<k<j$. For any $\mathbf{z} \in \mathcal{V}$ we have again $|\mathbf{r z}| \leq \alpha$, thus $\lfloor\mathbf{r z}+\alpha\rfloor \in\{0,1\}$ and $\tau_{\mathbf{r}, \alpha}(\mathbf{z}) \in \mathcal{V}$. Therefore, we have $\tau_{\mathbf{r}, \alpha}^{n}(\mathbf{z})=(-1,0, \ldots, 0)$ for some $n \geq 0$, hence $\tau_{\mathbf{r}, \alpha}^{n+1}(\mathbf{z})=\mathbf{0}$.

3. In this case we have $\mathcal{V}=\{-1,0,1\}^{d}$. As above, all periodic points of $\tau_{\mathbf{r}, \alpha}$ are in $\{0,-1\}^{d}$. If $\mathbf{z}=\left(z_{0}, z_{1}, \ldots, z_{d-1}\right)$ is a periodic point with $z_{d}=-\lfloor\mathbf{r z}+\alpha\rfloor=-1$, then we must have $z_{d-k}=-1$, and consequently $z_{d-j k}=-1$ for all $1 \leq j \leq d / k$. Then $z_{d}=-1$ also implies that $-\sum_{1 \leq j \leq d / k} r_{d-j k}+\alpha \geq 1$, i.e., (2) does not hold. On the other hand, if (2) holds, then the vector $\left(z_{0}, z_{1}, \ldots, z_{d-1}\right)$ with $z_{d-j k}=-1$ for $1 \leq j \leq d / k, z_{i}=0$ otherwise, is a periodic point of $\tau_{\mathbf{r}, \alpha}$.

Next we prove Property $(-\mathrm{F})$ when $\beta$ is a root of a polynomial with alternating coefficients, where the second highest coefficient is dominant.

Proof of Theorem 4. Let $\beta>1$ be a root of $p(x)=x^{d}-a_{1} x^{d-1}+a_{2} x^{d-2}+\cdots+(-1)^{d} a_{d} \in$ $\mathbb{Z}[x]$ with $a_{i} \geq 0$ for $i=1, \ldots, d$, and $a_{1} \geq 2+\sum_{i=2}^{d} a_{i}$. As $\frac{\mathrm{d}}{\mathrm{d} x}\left(p(x) x^{-d}\right) \geq \frac{a_{1}}{x^{2}}-\frac{a_{1}-2}{x^{3}}>0$ for $x>1$, the polynomial $p(x)$ has a unique $\operatorname{root} \beta>1$, and we have $\beta>a_{1}-1$ since $p\left(a_{1}-1\right) \leq-\left(a_{1}-1\right)^{d-1}+\left(a_{1}-2\right)\left(a_{1}-1\right)^{d-2}<0$. By Proposition [5, Property $(-\mathrm{F})$ holds if and only if $\left(r_{0}, r_{1}, \ldots, r_{d-2}\right) \in \mathcal{D}_{d-1, \alpha}^{0}$, with $r_{i}=a_{d-i} \beta^{-1}-a_{d-i+1} \beta^{-2}+a_{d-i+2} \beta^{-3}-\cdots+$ $(-1)^{d-i} a_{d} \beta^{-d+i-1}$. We have

$$
-\sum_{r_{i}<0} r_{i} \leq \frac{a_{1}-2}{\beta^{2}}+\frac{a_{1}-2}{\beta^{4}}+\cdots+\frac{a_{1}-2}{\beta^{2\lceil d / 2\rceil-2}} \leq \frac{a_{1}-2}{\beta^{2}-1}<\frac{1}{\beta+1}
$$


and

$$
\begin{aligned}
& \frac{\beta+1}{\beta} \sum_{i=0}^{d-1}\left|r_{i}\right| \leq \frac{\beta+1}{\beta}\left(\frac{a_{2}+\cdots+a_{d}}{\beta}+\frac{a_{3}+\cdots+a_{d}}{\beta^{2}}+\cdots+\frac{a_{d}}{\beta^{d-1}}\right) \\
& =\frac{a_{2}+\cdots+a_{d}}{\beta}+\frac{a_{2}+2 a_{3}+\cdots+2 a_{d}}{\beta^{2}}+\frac{a_{3}+2 a_{4}+\cdots+2 a_{d}}{\beta^{3}}+\cdots+\frac{a_{d-1}+2 a_{d}}{\beta^{d-1}}+\frac{a_{d}}{\beta^{d}} \\
& \leq \frac{a_{1}-2}{\beta}+\frac{2\left(a_{1}-2\right)-a_{2}}{\beta^{2}}+\frac{2\left(a_{1}-a_{2}-2\right)-a_{3}}{\beta^{3}}+\cdots+\frac{2\left(a_{1}-a_{2}-\cdots-a_{d-1}-2\right)-a_{d}}{\beta^{d}} \\
& \leq 1-2\left(\frac{1}{\beta}-\frac{a_{1}-2}{\beta^{2}}-\frac{a_{1}-a_{2}-2}{\beta^{3}}-\cdots-\frac{a_{1}-a_{2}-\cdots-a_{d-1}-2}{\beta^{d}}\right) \\
& \leq 1-\frac{2}{\beta}\left(1-\frac{a_{1}-2}{\beta-1}\right)<1 .
\end{aligned}
$$

Therefore, item 1 of Proposition 7 gives that Property $(-F)$ holds.

Now we can classify the cubic Pisot units with Property $(-F)$. The following description of cubic Pisot numbers in terms of the coefficients of the minimal polynomial is due to Akiyama [1, Lemma 1].

Lemma 8. A number $\beta>1$ with minimal polynomial $x^{3}-a x^{2}+b x-c$ is Pisot if and only if

$$
|b+1|<a+c \quad \text { and } \quad b+c^{2}<\operatorname{sgn}(c)(1+a c) .
$$

Proof of Theorem Q Let $\beta>1$ be a cubic Pisot unit with minimal polynomial $x^{3}-a x^{2}+$ $b x-c$. If $c=-1$, then $\beta$ has a negative conjugate, which contradicts Property $(-\mathrm{F})$ by [20]. Therefore, we assume in the following that $c=1$. Then from Lemma 8 we have that $-a-1 \leq b<a$. By Proposition 5 , Property $(-\mathrm{F})$ holds if and only if $\left(r_{0}, r_{1}\right) \in \mathcal{D}_{2, \alpha}^{0}$, with $\left(r_{0}, r_{1}\right)=\left(\frac{1}{\beta}, \frac{b}{\beta}-\frac{1}{\beta^{2}}\right)$ and $\alpha=\frac{\beta}{\beta+1}$. We distinguish five cases for the value of $b$.

1. $b=0$ : If $a \geq 2$, then we have $\left|r_{0}\right|+\left|r_{1}\right|=\frac{1}{\beta}+\frac{1}{\beta^{2}}<\alpha$ and $r_{0}+r_{1}>0>\alpha-1$, so we apply item 3 of Proposition [7. If $a=1$, then we have $T_{-\beta}^{-1}(0)=\{0\}$ as $\beta<\frac{1+\sqrt{5}}{2}$, thus $\operatorname{Fin}(-\beta)=\{0\}$.

2. $b=-1$ : If $a \geq 1$, then $r_{0}+r_{1}=-\frac{1}{\beta^{2}}>\frac{-1}{\beta+1}=\alpha-1$. If $a \geq 3$, then we also have $\left|r_{0}\right|+\left|r_{1}\right|<\alpha$ and use item 3 of Proposition 7. If $a=2$, then $r_{0} \approx 0.39, r_{1} \approx-0.55$, $\alpha \approx 0.72,\{-1,0,1\}^{2}$ is a set of witnesses, and Property $(-\mathrm{F})$ holds because $\tau_{\mathbf{r}, \alpha}$ acts on this set in the following way:

$$
\begin{gathered}
(-1,1) \mapsto(1,1) \mapsto(1,0) \mapsto(0,-1) \mapsto(-1,-1) \mapsto(-1,0) \mapsto(0,0), \\
(0,1) \mapsto(1,0), \quad(1,-1) \mapsto(-1,-1) .
\end{gathered}
$$

For $a=1$, we refer to Theorem 3, which is proved below. If $a=0$, then $\beta<\frac{1+\sqrt{5}}{2}$ and thus $\operatorname{Fin}(-\beta)=\{0\}$. 
3. $1 \leq b \leq a-2$ : For $b \geq 2$, we have $0<r_{0}<r_{1}<\alpha$ and thus $\left(r_{0}, r_{1}\right) \in \mathcal{D}_{2, \alpha}^{0}$ by item 2 of Proposition 7. If $b=1$, then we can use item 1 of Proposition 7 because $r_{0}, r_{1}>0$ and $r_{0}+r_{1}<\alpha$.

4. $1 \leq b=a-1$ : We have $\beta=b+\frac{1}{\beta(\beta-1)}$. For $b \geq 3$, we have $0<r_{0}<\alpha<r_{1}<1$, the set $\{-1,0,1\}^{2} \backslash\{(1,1),(-1,-1)\}$ is a set of witnesses, and $\tau_{\mathbf{r}, \alpha}$ acts on this set by

$$
(1,0) \mapsto(0,-1) \mapsto(-1,1) \mapsto(1,-1) \mapsto(-1,0) \mapsto(0,0), \quad(0,1) \mapsto(1,-1),
$$

thus Property $(-\mathrm{F})$ holds. If $b=2$, then $0<r_{0}<r_{1}<\alpha$ and we can use item 2 of Proposition 7 . If $b=1$, then $r_{0} \approx 0.57, r_{1} \approx 0.25, \alpha \approx 0.64$, thus $\{-1,0,1\}^{2}$ is a set of witnesses, with

$$
\begin{gathered}
(-1,-1) \mapsto(-1,1) \mapsto(1,0) \mapsto(0,-1) \mapsto(-1,0) \mapsto(0,0), \\
(0,1) \mapsto(1,0),(1,1) \mapsto(1,-1) \mapsto(-1,0) .
\end{gathered}
$$

5. $-a-1 \leq b \leq-2$ : We have $-r_{0}-r_{1}+\alpha=\frac{-b-1}{\beta}+\frac{1}{\beta^{2}}+\frac{\beta}{\beta+1}>1$, thus $\tau_{\mathbf{r}, \alpha}(-1,-1)=$ $(-1,-1)$, hence $\left(r_{0}, r_{1}\right) \notin \mathcal{D}_{2, \alpha}^{0}$.

Therefore, $\beta$ has Property (-F) if and only if $-1 \leq b<a,|a|+|b| \geq 2$.

Finally, we study generalized $d$-bonacci numbers.

Proof of Theorem [3. Let $\beta>1$ be a root of $x^{d}-m x^{d-1}-\cdots-m x-m$ with $d, m \in \mathbb{N}$.

If $d=1$ (and $m \geq 2$ ), then $\beta$ is an integer, and Property $(-\mathrm{F})$ follows from $\mathbb{Z}_{-\beta}=\mathbb{Z}$; see e.g. [20].

If $d=3$, then $\mathbf{r}=\left(\frac{m}{\beta},-\frac{m}{\beta}-\frac{m}{\beta^{2}}\right), 0<r_{0}<\alpha<-r_{1}<1$, with $\alpha=\frac{\beta}{\beta+1}$, and $\tau_{\mathbf{r}, \alpha}$ satisfies

$$
(0,1) \mapsto(1,1) \mapsto(1,0) \mapsto(0,-1) \mapsto(-1,-1) \mapsto(-1,0) \mapsto(0,0),
$$

with $\{-1,0,1\}^{2} \backslash\{(1,-1),(-1,1)\}$ being a set of witnesses.

If $d=5$, then $\mathbf{r}=\left(\frac{m}{\beta},-\frac{m}{\beta}-\frac{m}{\beta^{2}}, \frac{m}{\beta}+\frac{m}{\beta^{2}}+\frac{m}{\beta^{3}},-\frac{m}{\beta}-\frac{m}{\beta^{2}}-\frac{m}{\beta^{3}}-\frac{m}{\beta^{4}}\right)$, which gives $0<r_{0}<$ $\alpha<-r_{1}<r_{2}<-r_{3}<1$ and the $\tau_{\mathbf{r}, \alpha}$-transitions

$$
\begin{gathered}
(0,1,0,0) \mapsto(1,0,0,1) \mapsto(0,0,1,0) \mapsto(0,1,0,-1) \mapsto(1,0,-1,0) \mapsto(0,-1,0,0) \mapsto \\
(-1,0,0,-1) \mapsto(0,0,-1,-1) \mapsto(0,-1,-1,0) \mapsto(-1,-1,0,0) \mapsto(-1,0,0,0) \mapsto(0,0,0,0), \\
(0,0,-1,0) \mapsto(0,-1,0,1) \mapsto(-1,0,1,0) \mapsto(0,1,0,-1), \\
(0,1,1,1) \mapsto(1,1,1,1) \mapsto(1,1,1,0) \mapsto(1,1,0,-1) \mapsto(1,0,-1,-1) \mapsto \\
(0,-1,-1,-1) \mapsto(-1,-1,-1,-1) \mapsto(-1,-1,-1,0) \mapsto(-1,-1,0,0), \\
(0,0,0,1) \mapsto(0,0,1,1) \mapsto(0,1,1,0) \mapsto(1,1,0,0) \mapsto(1,0,0,0) \mapsto(0,0,0,-1) \mapsto(0,0,-1,-1), \\
(-1,-1,0,1) \mapsto(-1,0,1,1) \mapsto(0,1,1,0) .
\end{gathered}
$$


Let $\mathcal{V}$ be the set of these states. We have $\pm \mathbf{e}_{i} \in \mathcal{V}, \mathbf{z} \in \mathcal{V}$ if and only if $-\mathbf{z} \in \mathcal{V}$ and $\tau_{\mathbf{r}, 0}(\mathbf{z}) \in \mathcal{V}$ for all $\mathbf{z} \in \mathcal{V}$, thus $\mathcal{V}$ is a set of witnesses. As $\tau_{\mathbf{r}, \alpha}^{11}(\mathbf{z})=(0,0,0,0)$ for all $\mathbf{z} \in \mathcal{V}$, $\beta$ has Property $(-\mathrm{F})$.

For odd $d \geq 7$, Property $(-\mathrm{F})$ does not hold since $T_{-\beta}^{d-1}\left(\frac{m}{\beta^{2}}+\frac{m}{\beta^{3}}+\frac{m}{\beta^{4}}-1\right)=\frac{m}{\beta^{2}}+\frac{m}{\beta^{3}}+\frac{m}{\beta^{4}}-1$, i.e., $\tau_{\mathbf{r}, \alpha}^{d-1}(-1,0,0,-1,0,0, \ldots, 0)=(-1,0,0,-1,0,0, \ldots, 0)$. For even $d \geq 2$, we use the second condition of Theorem 1, or that $\tau_{\mathbf{r}, \alpha}(-1, \ldots,-1)=(-1, \ldots,-1)$.

Therefore, $\beta$ has Property $(-\mathrm{F})$ if and only if $d \in\{1,3,5\}$.

\section{Addition and subtraction}

In this section, we consider the lengths of fractional parts arising in the addition and subtraction of $(-\beta)$-integers; we prove the following theorem.

Theorem 9. Let $\beta>1$ be a root of $x^{3}-m \beta^{2}-m \beta-m, m \geq 1$. We have

$$
\max \left\{\operatorname{fr}(x \pm y): x, y \in \mathbb{Z}_{-\beta}\right\}=3 m+ \begin{cases}3 & \text { if } m=1 \text { or } m \text { is even } \\ 4 & \text { if } m \geq 3 \text { is odd. }\end{cases}
$$

Throughout the section, let $\beta$ be as in Theorem 9, $\mathbf{r}=\left(r_{0}, r_{1}\right)=\left(\frac{m}{\beta},-\frac{m}{\beta}-\frac{m}{\beta^{2}}\right)$ and $\alpha=\frac{\beta}{\beta+1}$. Recall that $x, y \in \mathbb{Z}_{-\beta}$ means that $T_{-\beta}^{k}\left(\frac{x}{(-\beta)^{k}}\right)=0=T_{-\beta}^{k}\left(\frac{y}{(-\beta)^{k}}\right)$, and $\operatorname{fr}(x \pm y)=n$ is the minimal $n \geq 0$ such that $T_{-\beta}^{k+n}\left(\frac{x \pm y}{(-\beta)^{k}}\right)=0$, with $k \geq 0$ such that $\frac{x}{(-\beta)^{k}}, \frac{y}{(-\beta)^{k}}, \frac{x \pm y}{(-\beta)^{k}} \in$ $\left(\ell_{\beta}, \ell_{\beta}+1\right)$. To determine $\operatorname{fr}(x-y)$, set

$$
s_{j}=T_{-\beta}^{j}\left(\frac{x-y}{(-\beta)^{k}}\right)+T_{-\beta}^{j}\left(\frac{y}{(-\beta)^{k}}\right)-T_{-\beta}^{j}\left(\frac{x}{(-\beta)^{k}}\right)
$$

for $j \geq 0$. Then we have $s_{j}=T_{-\beta}^{j}\left(\frac{x-y}{(-\beta)^{k}}\right)$ for $j \geq k$, and, for all $j \geq 0$,

$$
\begin{gathered}
s_{j+1} \in-\beta s_{j}+\mathcal{B} \quad \text { with } \quad \mathcal{B}=-\mathcal{A}-\mathcal{A}+\mathcal{A}=\{-2 m,-2 m+1, \ldots, m\}, \\
s_{j} \in\left[\ell_{\beta}, \ell_{\beta}+1\right)+\left[\ell_{\beta}, \ell_{\beta}+1\right)-\left[\ell_{\beta}, \ell_{\beta}+1\right)=\left(\ell_{\beta}-1, \ell_{\beta}+2\right) .
\end{gathered}
$$

As $s_{0}=0$, we have $s_{j} \in \mathbb{Z}[\beta]$ for $j \geq 0$. Therefore, we extend the bijection $\phi: \mathbb{Z}^{2} \rightarrow$ $\mathbb{Z}[\beta] \cap\left[\ell_{\beta}, \ell_{\beta}+1\right)$ to

$$
\Phi: \mathbb{Z}^{2} \times\{-1,0,1\} \rightarrow \mathbb{Z}[\beta] \cap\left[\ell_{\beta}-1, \ell_{\beta}+2\right), \quad(\mathbf{z}, h) \mapsto \mathbf{r z}-\lfloor\mathbf{r z}+\alpha\rfloor+h .
$$

Note that $\Phi(\mathbf{z}, 0)=\phi(\mathbf{z})$.

Lemma 10. Let $\mathbf{z}=\left(z_{0}, z_{1}\right) \in \mathbb{Z}^{2}, h \in\{-1,0,1\}$ and $b \in \mathcal{B}$. Then

$-\beta \Phi(\mathbf{z}, h)+b=\Phi\left(z_{1}, h-\lfloor\mathbf{r z}+\alpha\rfloor,\left(z_{1}-z_{0}-h+\lfloor\mathbf{r z}+\alpha\rfloor\right) m+\left\lfloor r_{0} z_{1}+r_{1} h-r_{1}\lfloor\mathbf{r z}+\alpha\rfloor+\alpha\right\rfloor+b\right)$. 
Proof. We have

$$
\begin{aligned}
-\beta \Phi(\mathbf{z}, h)+b & =-z_{0} m+z_{1} m+\frac{z_{1} m}{\beta}+\lfloor\mathbf{r z}+\alpha\rfloor \beta-h \beta+b \\
& =r_{0} z_{1}+r_{1}(h-\lfloor\mathbf{r z}+\alpha\rfloor)+\left(z_{1}-z_{0}-h+\lfloor\mathbf{r z}+\alpha\rfloor\right) m+b .
\end{aligned}
$$

Hence, we have $s_{j} \in \Phi\left(\tilde{\tau}_{\mathbf{r}, \alpha}^{j}(\mathbf{0}, 0)\right)$, where $\tilde{\tau}_{\mathbf{r}, \alpha}$ extends $\tau_{\mathbf{r}, \alpha}$ to a set-valued function by

$$
\begin{aligned}
& \tilde{\tau}_{\mathbf{r}, \alpha}: \mathbb{Z}^{2} \times\{-1,0,1\} \rightarrow \mathcal{P}\left(\mathbb{Z}^{2} \times\{-1,0,1\}\right), \quad(\mathbf{z}, h) \mapsto\left\{\left(z_{1}, h-\lfloor\mathbf{r z}+\alpha\rfloor, h^{\prime}\right):\right. \\
& \left.h^{\prime} \in\{-1,0,1\} \cap\left(\left(z_{1}-z_{0}-h+\lfloor\mathbf{r z}+\alpha\rfloor\right) m+\left\lfloor r_{0} z_{1}+r_{1} h-r_{1}\lfloor\mathbf{r z}+\alpha\rfloor+\alpha\right\rfloor+\mathcal{B}\right)\right\} .
\end{aligned}
$$

To give a bound for the sets $\tilde{\tau}_{\mathbf{r}, \alpha}^{j}(\mathbf{0}, 0)$, let

$$
\begin{gathered}
A_{k}=\{(j, k):-1 \leq j<k\}, B_{k}=\{(k, j): 1 \leq j \leq k\}, C_{k}=\{(j, j-k): 1 \leq j \leq k\}, \\
D_{k}=\{(-j,-k): 0 \leq j<k\}, E_{k}=\{(-k,-j): 2 \leq j \leq k\}, \\
F_{k}=\{(-j, k-j): 2 \leq j \leq k+1\} .
\end{gathered}
$$

Then $\bigcup_{k>0}\left\{A_{k}, B_{k}, C_{k}, D_{k}, E_{k}, F_{k}\right\}$ forms a partition of $\mathbb{Z}^{2} \backslash\{(0,0),(-1,-1)\}$, with the sets $B_{0}, C_{0}, D_{0}, E_{0}, F_{0}$, and $E_{1}$ being empty, see Figure 1. If $m \geq 2$, then let

$$
\begin{aligned}
V & =\left(\bigcup_{0 \leq k \leq m}\left(A_{k} \cup B_{k} \cup C_{k} \cup D_{k} \cup E_{k} \cup F_{k}\right) \times\{-1,0,1\}\right) \backslash\{(-1, m, 1),(0, m, 1)\} \\
& \cup\left(\left(C_{m+1} \backslash\{(m+1,0)\}\right) \times\{1\}\right) \cup\left(D_{m+1} \times\{1\}\right) \cup\left(\left(D_{m+1} \backslash\{(0,-m-1)\}\right) \times\{0\}\right) \\
& \cup\left(D_{m+1} \backslash\{(0,-m-1),(-1,-m-1),(-2,-m-1)\}\right) \times\{-1\} \\
& \cup\left(\left(\{(0,0),(-1,-1)\} \cup E_{m+1}\right) \backslash\{(-m-1,-m-1)\}\right) \times\{-1,0,1\} \\
& \cup\left(\left(F_{m+1} \backslash\{(-m-2,-1),(-m-1,0)\}\right) \times\{-1,0\}\right) .
\end{aligned}
$$

If $m=1$, then we add the point $(-2,0,-1)$ to this set, i.e.,

$$
\begin{gathered}
V=(\{(0,0),(1,1),(1,0),(0,-1),(-1,-1),(-1,0),(-2,-1)\} \times\{-1,0,1\}) \\
\cup(\{(-1,1),(0,1)\} \times\{-1,0\}) \cup(\{(-1,-2)\} \times\{0,1\}) \cup\{(1,-1,1),(0,-2,1),(-2,0,-1)\} .
\end{gathered}
$$

We call a point $\mathbf{z} \in \mathbb{Z}^{2}$ full if $\{\mathbf{z}\} \times\{-1,0,1\} \subset V$.

The following result is the key lemma of this section.

Lemma 11. Let $x, y \in\left[\ell_{\beta}, \ell_{\beta}+1\right)$ such that $x-y \in\left[\ell_{\beta}, \ell_{\beta}+1\right)$. Then $T_{-\beta}^{j}(x-y)+$ $T_{-\beta}^{j}(y)-T_{-\beta}^{j}(x) \in \Phi(V)$ for all $j \geq 0$.

To prove Lemma 11, we first determine the value of $\lfloor\mathbf{r z}+\alpha\rfloor$ for $(\mathbf{z}, h) \in V$. 


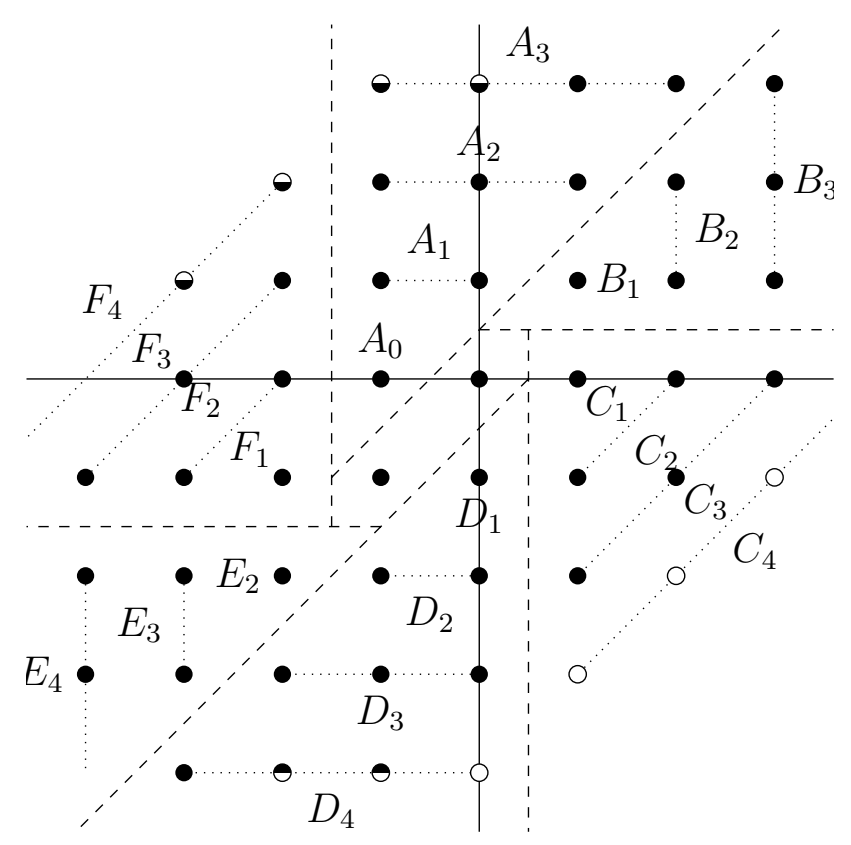

Figure 1: The set $V$ for $m=3$. Full points are represented by disks, points $\mathbf{z}$ with $\{\mathbf{z}\} \times\{0,1\} \subset V,\{\mathbf{z}\} \times\{-1,0\} \subset V$ and $\{\mathbf{z}\} \times\{1\} \subset V$ by upper half-disks, lower half-disks and circles respectively.

Lemma 12. Let $\mathbf{z}=\left(z_{0}, z_{1}\right) \in \mathbb{Z}^{2}$ with $-m-1 \leq z_{0} \leq m,\left|z_{1}\right| \leq m+1$ and $\left|z_{0}-z_{1}\right| \leq m+1$. Then

$$
\lfloor\mathbf{r z}+\alpha\rfloor=z_{0}-z_{1}+ \begin{cases}0 & \text { if } z_{0} \geq 0 \text { or } z_{1} \leq z_{0}=-1 \\ 1 & \text { if } z_{0} \leq-2 \text { or } z_{1}>z_{0}=-1\end{cases}
$$

Proof. We have $z_{0} r_{0}+z_{1} r_{1}=z_{0}-z_{1}-z_{0} \frac{m}{\beta^{2}}+\left(z_{1}-z_{0}\right) \frac{m}{\beta^{3}}$ and

$\frac{-\beta}{\beta+1}<-\frac{m^{2}}{\beta^{2}}-\frac{(m+1) m}{\beta^{3}} \leq-\frac{z_{0} m}{\beta^{2}}+\frac{\left(z_{1}-z_{0}\right) m}{\beta^{3}} \leq \frac{(m+1) m}{\beta^{2}}+\frac{(m+1) m}{\beta^{3}}<1+\frac{1}{\beta+1}$, thus $\lfloor\mathbf{r z}+\alpha\rfloor \in z_{0}-z_{1}+\{0,1\}$.

If $z_{0} \geq 0$, then we have $-z_{0} \frac{m}{\beta^{2}}+\left(z_{1}-z_{0}\right) \frac{m}{\beta^{3}} \leq(m+1) \frac{m}{\beta^{3}}<\frac{1}{\beta+1}$. If $z_{1} \leq z_{0}=-1$, then $-z_{0} \frac{m}{\beta^{2}}+\left(z_{1}-z_{0}\right) \frac{m}{\beta^{3}} \leq \frac{m}{\beta^{2}}<\frac{1}{\beta+1}$. This shows that $\lfloor\mathbf{r z}+\alpha\rfloor=z_{0}-z_{1}$ in these two cases.

If $z_{1}>z_{0}=-1$, then we have $-z_{0} \frac{m}{\beta^{2}}+\left(z_{1}-z_{0}\right) \frac{m}{\beta^{3}} \geq \frac{m}{\beta^{2}}+\frac{m}{\beta^{3}}>\frac{1}{\beta+1}$. Finally, if $z_{0} \leq-2$, then $-z_{0} \frac{m}{\beta^{2}}+\left(z_{1}-z_{0}\right) \frac{m}{\beta^{3}} \geq 2 \frac{m}{\beta^{2}}-(m-1) \frac{m}{\beta^{3}}>\frac{1}{\beta+1}$, thus $\lfloor\mathbf{r z}+\alpha\rfloor=z_{0}-z_{1}+1$ in the latter cases.

Proof of Lemma 11. We have already seen above that $T_{-\beta}^{j}(x-y)+T_{-\beta}^{j}(y)-T_{-\beta}^{j}(x) \in$ $\Phi\left(\tilde{\tau}_{\mathbf{r}, \alpha}^{j}(\mathbf{0}, 0)\right)$. As $(\mathbf{0}, 0) \in V$, it suffices to show that $\tilde{\tau}_{\mathbf{r}, \alpha}(V) \subseteq V$.

Let $(\mathbf{z}, h) \in V$ and $b \in B$ such that

$$
h^{\prime}=\left(z_{1}-z_{0}+\lfloor\mathbf{r z}+\alpha\rfloor-h\right) m+\left\lfloor r_{0} z_{1}+r_{1} h-r_{1}\lfloor\mathbf{r z}+\alpha\rfloor+\alpha\right\rfloor+b \in\{-1,0,1\},
$$


i.e., $\left(z_{1}, h-\lfloor\mathbf{r z}+\alpha\rfloor, h^{\prime}\right) \in \tilde{\tau}_{\mathbf{r}, \alpha}(\mathbf{z}, h)$. If $\left(z_{1}, h-\lfloor\mathbf{r z}+\alpha\rfloor\right)$ is full, then we clearly have $\tilde{\tau}_{\mathbf{r}, \alpha}(\mathbf{z}, h) \subset V$. Otherwise, we have to consider the possible values of $h^{\prime}$. We distinguish seven cases.

1. $\mathbf{z} \in\{(0,0),(-1,-1)\}:$ We have $\lfloor\mathbf{r z}+\alpha\rfloor=0$.

If $m \geq 2$, then $(0, h)$ and $(-1, h)$ are full since $(0,-1) \in D_{1},(0,1),(-1,1) \in A_{1}$, $(-1,0) \in A_{0}$, and $(0,0),(-1,-1)$ are also full.

If $m=1$, then $(0,-1),(0,0),(-1,-1),(-1,0)$ are full. For $h=1$, we have $h^{\prime}=$ $-1+\left\lfloor r_{0} z_{1}+r_{1}+\alpha\right\rfloor+b=b-2$, thus $h^{\prime}=1$. The points $\left(z_{1}, 1,-1\right)$ for $z_{1} \in\{-1,0\}$ are in $V$.

2. $\mathbf{z}=(j, k) \in A_{k}$ : We have $\lfloor\mathbf{r z}+\alpha\rfloor=-k$ for $j=-1$ and $\lfloor\mathbf{r z}+\alpha\rfloor=j-k$ for $0 \leq j<k$.

If $k=0$, then $\mathbf{z}=(-1,0)$, and $(0, h)$ is full for $m \geq 2$. If $m=1$, then $(0,0)$ and $(0,-1)$ are full, and $h=1$ gives that $h^{\prime}=\left\lfloor r_{1}+\alpha\right\rfloor+b=b-1 \in\{-1,0\}$, thus $\tilde{\tau}_{\mathbf{r}, \alpha}(\mathbf{z}, 1) \subset V$.

If $1 \leq k<m$, then $(k, h+k),(k, h-j+k)$ lie in $B_{k} \cup C_{k} \cup\{(k, k+1)\}$ and are full. If $k=m$, then we have either $h \in\{-1,0\}$, thus $(m, h+m)$ and $(m, h-j+m)$ lie in the set of full points $B_{m} \cup C_{m}$, or $h=1$ and $1 \leq j<m$, in which case $(m, 1-j+m) \in B_{m}$ is also full. (Note that $(-1, m, 1),(0, m, 1) \notin V$.)

3. $\mathbf{z}=(k, j) \in B_{k}$ : We have $\lfloor\mathbf{r z}+\alpha\rfloor=k-j, 1 \leq j \leq k$.

For $h \in\{0,1\}$, the point $(j, h+j-k)$ is in $C_{k}$ and $C_{k-1} \cup B_{k}$ respectively, hence full. The point $(j, j-k-1) \in C_{k+1}$ is full if $k<m$. Finally, if $k=m$ and $h=-1$, then $h^{\prime}=m+\left\lfloor r_{0} j+r_{1}(j-m-1)+\alpha\right\rfloor+b=2 m+1+b=1$, and $(j, j-m-1,1) \in V$.

4. $\mathbf{z}=(j, j-k) \in C_{k}$ : We have $\lfloor\mathbf{r z}+\alpha\rfloor=k, 1 \leq j \leq k$.

The point $(j-k, h-k), h \in\{-1,0,1\}$, is in $D_{k+1}, D_{k}$, and $D_{k-1} \cup E_{k-1} \cup$ $\{(0,0),(-1,-1)\}$ respectively, hence full for all $k<m, k \leq m$, and $k \leq m+1$ respectively. It remains to consider $h=-1, k=m$. For $1 \leq j \leq m-3$, the point $(j-m,-m-1)$ is full; we have

$h^{\prime}=m+\left\lfloor r_{0}(j-m)-r_{1}(m+1)+\alpha\right\rfloor+b= \begin{cases}2 m+1+b=1 & \text { if } j=m, \\ 2 m+b \in\{0,1\} & \text { if } j \in\{m-2, m-1\},\end{cases}$ $(0,-m-1,1) \in V$, and $\{(j-m,-m-1)\} \times\{0,1\} \subset V$ for $\max (1, m-2) \leq j<m$.

5. $\mathbf{z}=(-j,-k) \in D_{k}$ : We have $\lfloor\mathbf{r z}+\alpha\rfloor=k-j$ if $j \in\{0,1\},\lfloor\mathbf{r z}+\alpha\rfloor=k-j+1$ if $2 \leq j<k$.

Let first $k=1$, i.e., $\mathbf{z}=(0,-1)$. The point $(-1, h-1)$ lies in $D_{2} \cup\{(-1,-1)\} \cup A_{0}$ and is full, except if $m=1, h=-1$; in the latter case, we have $h^{\prime}=1+\left\lfloor-r_{0}-2 r_{1}+\alpha\right\rfloor+b=$ $b+2 \in\{0,1\}$, and $\{(-1,-2)\} \times\{0,1\} \in V$. 
For $2 \leq k \leq m$, the points $(-k, h+j-k), j \in\{0,1\}$, and $(-k, h+j-k-1)$, $2 \leq j<k$, lie in $\{(-k,-i): 0 \leq i \leq k+1\}$, and are full, except for $k=m=2$, $h=-1, j=0$; in the latter case, we have $h^{\prime}=2+\left\lfloor-2 r_{0}-3 r_{1}+\alpha\right\rfloor+b=b+4 \in\{0,1\}$, and $\{(-2,-3)\} \times\{0,1\} \in V$.

Finally, for $k=m+1$, we have $j=0, h=1$, or $1 \leq j \leq \min (m, 2), h \in\{0,1\}$, or $3 \leq j \leq m, h \in\{-1,0,1\}$, thus the points $(-m-1, h+j-m-1), j \in\{0,1\}$, and $(-m-1, h+j-m-2), 2 \leq j \leq m$, lie in $\{(-m-1,-i): \min (m-1,1) \leq i \leq m\}$ and are full, except for $m=j=h=1$; in the latter case, we have $h^{\prime}=-1+\left\lfloor-2 r_{0}+\right.$ $\alpha\rfloor+b=b-2=-1$, and $(-2,0,-1) \in V$.

6. $\mathbf{z}=(-k,-j) \in E_{k}$ : We have $\lfloor\mathbf{r z}+\alpha\rfloor=j-k+1,2 \leq j \leq k$.

The point $(-j, h-j+k-1) \in F_{k-2} \cup F_{k-1} \cup F_{k} \cup\{(-k,-2)\}$ is full, except for $k=m+1$, $h=1$; in the latter case, we have $2 \leq j \leq m, h^{\prime}=\left\lfloor-r_{0} j+r_{1}(m-j+1)+\alpha\right\rfloor+b=$ $b-m \in\{-1,0\}$, and $\{(-j, m-j+1)\} \times\{-1,0\} \subset V$.

7. $\mathbf{z}=(-j, k-j) \in F_{k}$ : We have $\lfloor\mathbf{r z}+\alpha\rfloor=1-k, 2 \leq j \leq k+1$.

If $1 \leq k \leq m$, then the point $(k-j, h+k-1) \in A_{k-2} \cup A_{k-1} \cup A_{k} \cup\{(k-2, k-2)\}$ is full, except for $k=m, j \in\{m, m+1\}, h=1$; in the latter case, we have $h^{\prime}=\left\lfloor r_{0}(m-j)+r_{1} m+\alpha\right\rfloor+b=b-m \in\{-1,0\}$, and $\{(m-j, m)\} \times\{-1,0\} \subset V$. If $k=m+1$, then $2 \leq j \leq m, h \in\{-1,0\}$, or $m=1, j=2, h=-1$, and $(m+1-j, h+m) \in A_{m-1} \cup A_{m} \cup\{(m-1, m-1)\}$ is full.

Lemma 13. For the following chains of sets, $\tau_{\mathbf{r}, \alpha}$ maps elements of a set into its successor:

$$
\begin{aligned}
C_{k} \backslash\{(m+1,0)\} & \rightarrow D_{k} \rightarrow E_{k} \rightarrow F_{k-1} \quad(3 \leq k \leq m+1), \\
F_{k+1} \rightarrow A_{k} & \rightarrow B_{k} \rightarrow C_{k} \rightarrow D_{k} \quad(1 \leq k \leq m) .
\end{aligned}
$$

On the remaining $\mathbf{z}=\left(z_{0}, z_{1}\right) \in \mathbb{Z}^{2}$ with $-m-1 \leq z_{0} \leq m,-m-1 \leq z_{1} \leq m$ and $\left|z_{0}-z_{1}\right| \leq m+1, \tau_{\mathbf{r}, \alpha}$ acts by

$$
\begin{aligned}
(0,-2) & \mapsto(-2,-2) \mapsto(-2,-1) \mapsto(-1,0) \\
(-1,-2) & \mapsto(-2,-1), \quad(0,-1) \mapsto(-1,-1)
\end{aligned}
$$

Proof. This is a direct consequence of Lemma 12, except for $(-m-2,-1) \in F_{m+1}$; see also the proof of Lemma 11. As $\frac{1}{\beta+1}<\frac{(m+2) m}{\beta^{2}}+\frac{(m+1) m}{\beta^{3}}<1+\frac{m}{\beta^{2}}<1+\frac{1}{\beta+1}$, the proof of Lemma 12 shows that $\tau_{\mathbf{r}, \alpha}(-m-2,-1)=(-1, m) \in A_{m}$.

Proposition 14. We have

$$
\max \left\{\operatorname{fr}(x-y): x, y \in \mathbb{Z}_{-\beta}\right\}=3 m+ \begin{cases}3 & \text { if } m=1 \text { or } m \text { is even } \\ 4 & \text { if } m \geq 3 \text { is odd }\end{cases}
$$


Proof. Let $k \geq 0$ be such that $\frac{x}{(-\beta)^{k}}, \frac{y}{(-\beta)^{k}}, \frac{x-y}{(-\beta)^{k}} \in\left(\ell_{\beta}, \ell_{\beta}+1\right)$. Then $\operatorname{fr}(x-y)=n$ is the minimal $n \geq 0$ such that $T_{-\beta}^{k+n}\left(\frac{x-y}{(-\beta)^{k}}\right)=0$. Let $\mathbf{z} \in \mathbb{Z}^{2}$ be such that $T_{-\beta}^{k}\left(\frac{x-y}{(-\beta)^{k}}\right)=\phi(\mathbf{z})$. Then $\operatorname{fr}(x-y)$ is the minimal $n \geq 0$ such that $\tau_{\mathbf{r}, \alpha}^{n}(\mathbf{z})=0$, and we have $(\mathbf{z}, 0) \in V$, i.e.,

$$
\begin{aligned}
\mathbf{z} \in\{(0,0),(-1,-1),(0,-1)\} \cup \bigcup_{0 \leq k \leq m}\left(A_{k} \cup B_{k} \cup C_{k} \cup D_{k+1} \cup E_{k+1} \cup F_{k+1}\right) \\
\qquad\{(0,-m-1),(-m-1,-m-1),(-m-2,-1),(-m-1,0)\} .
\end{aligned}
$$

Therefore, $\operatorname{fr}(x-y)$ is bounded by the maximal length of the path from $\mathbf{z}$ to $(0,0)$ given by Lemma 13 .

For $1 \leq k \leq m / 2$, the sets $F_{2 k+1}, A_{2 k}, B_{2 k}$, and $C_{2 k}$ are mapped to $D_{2}$ in $6 k-2,6 k-3$, $6 k-4$, and $6 k-5$ steps respectively. For $2 \leq k \leq(m+1) / 2$, the sets $D_{2 k}$ and $E_{2 k}$ are mapped to $D_{2}$ in $6 k-6$ and $6 k-7$ steps respectively. The points $(0,-2)$ and $(-1,-2)$ in $D_{2}$ are mapped to $(0,0)$ in 4 and 3 steps respectively.

Similarly, for $1 \leq k \leq(m+1) / 2$, the sets $F_{2 k}, A_{2 k-1}, B_{2 k-1}$, and $C_{2 k-1}$ are mapped to $D_{1}=\{(0,-1)\}$ in $6 k-2,6 k-3,6 k-4$, and $6 k-5$ steps respectively. For $1 \leq k \leq m / 2$, the sets $D_{2 k+1}$ and $E_{2 k+1}$ are mapped to $D_{1}$ in $6 k$ and $6 k-1$ steps respectively. Finally, the point $(0,-1) \in D_{1}$ is mapped to $(0,0)$ in 3 steps.

For even $m$, the longest path comes thus from $D_{m+1}$ and has length $3 m+3$. For odd $m \geq 3$, the longest path comes from $F_{m+1}$ and has length $3 m+4$. For $m=1$, the longest path comes from $A_{1}$ (since $F_{2} \times\{0\} \cap V=\emptyset$ in this case) and has length 6 . This proves the upper bound for $\operatorname{fr}(x-y)$.

For $m=1$, this bound is attained by $x=1-\beta, y=\beta^{4}-\beta^{3}$, since $\operatorname{fr}(x-y)=\operatorname{fr}\left(\frac{1}{\beta^{3}}-\right.$ $\left.\beta^{3}\right)=\operatorname{fr}\left(\frac{1}{\beta^{3}}\right)=6$. Assume in the following that $m \geq 2$. Then the points $(-m,-m-1,0) \in$ $D_{m+1} \times\{0\}$ and $(-2, m-1,0) \in F_{m+1} \times\{0\}$ are in $\tilde{\tau}_{\mathbf{r}, \alpha}^{j}(0,0,0)$ for sufficiently large $j$ because they can be attained from $(0,0,0)$ by transitions

$$
(\mathbf{z}, h) \stackrel{b}{\longrightarrow}\left(z_{1}, h-\lfloor\mathbf{r z}+\alpha\rfloor,\left(z_{1}-z_{0}+\lfloor\mathbf{r z}+\alpha\rfloor-h\right) m+\left\lfloor r_{0} z_{1}+r_{1} h-r_{1}\lfloor\mathbf{r z}+\alpha\rfloor+\alpha\right\rfloor+b\right)
$$

with $b \in B$ by the following paths (cf. the proof of Lemma 11):

$$
\begin{gathered}
(0, k, 0) \stackrel{-1}{\longrightarrow}(k, k,-1) \stackrel{-m-k-2}{\longrightarrow}(k,-1,-1) \stackrel{-m-k-2}{\longrightarrow}(-1,-k-2,-1), \quad(0 \leq k \leq m-2) \\
(-1,-k,-1) \stackrel{-m}{\longrightarrow}(-k,-k, 1) \stackrel{k}{\longrightarrow}(-k, 0,1) \stackrel{k}{\longrightarrow}(0, k, 0), \quad(2 \leq k \leq m) \\
(0, m-1,0) \stackrel{-1}{\longrightarrow}(m-1, m-1,-1) \stackrel{-2 m}{\longrightarrow}(m-1,-1,0) \stackrel{-m}{\longrightarrow}(-1,-m,-1), \\
(0, m, 0) \stackrel{0}{\longrightarrow}(m, m, 0) \stackrel{-m}{\longrightarrow}(m, 0,0) \stackrel{-m-1}{\longrightarrow}(0,-m,-1), \\
(0,-m,-1) \stackrel{-m-2}{\longrightarrow}(-m,-m-1,0) \stackrel{-1}{\longrightarrow}(-m-1,-2,1) \stackrel{m}{\longrightarrow}(-2, m-1,0) .
\end{gathered}
$$

For even $m \geq 2$, these paths correspond to

$$
\begin{aligned}
& \operatorname{fr}\left(000022000044 \cdots 0000 m m 0000 \bullet 0^{\omega}-022000044000 \cdots 0 m m 0000012 \bullet 0^{\omega}\right) \\
& \quad=\operatorname{fr}\left((1 m m m 00)^{m / 2} 0 m m m \bullet d_{-\beta}(\phi(-m,-m-1))\right)=\operatorname{fr}(\phi(-m,-m-1))=3 m+3 ;
\end{aligned}
$$


for the second equality, we have used that $(1 m m m 00)^{m / 2} 0 m m m \bullet d_{-\beta}(\phi(-m,-m-1))$ is a $(-\beta)$-expansion. Indeed, this follows from the lexicographic conditions given in [17] since $d_{-\beta}\left(\ell_{\beta}\right)=m 0 m^{\omega}$ and $d_{-\beta}(\phi(-m,-m-1))$ starts with $2($ as $-\beta \phi(-m,-m-1)-2=$ $\phi(-m-1,-2))$. For odd $m \geq 3$, we have

$$
\begin{aligned}
& \operatorname{fr}\left(000022000044 \cdots 0000(m-1)(m-1) 0000 m m 00000 m \bullet 0^{\omega}\right. \\
& \left.\quad-022000044000 \cdots 0(m-1)(m-1) 0000 m 0000001200 \bullet 0^{\omega}\right) \\
& \quad=\operatorname{fr}\left((1 m m m 00)^{(m+1) / 2} 0 m m m 10 \bullet d_{-\beta}(\phi(-2, m-1))\right)=\operatorname{fr}(\phi(-2, m-1))=3 m+4 .
\end{aligned}
$$

This concludes the proof of the proposition.

Proposition 15. We have

$$
\max \left\{\operatorname{fr}(x+y): x, y \in \mathbb{Z}_{-\beta}\right\} \leq \max \left\{\operatorname{fr}(x-y): x, y \in \mathbb{Z}_{-\beta}\right\} .
$$

Proof. Let $\mu=\max \left\{\operatorname{fr}(x-y): x, y \in \mathbb{Z}_{-\beta}\right\}$. For $x, y \in \mathbb{Z}_{-\beta}, \operatorname{fr}(x+y)$ is the minimal $n \geq 0$ such that $T_{-\beta}^{k+n}\left(\frac{x+y}{(-\beta)^{k}}\right)=0$, with $k \geq 0$ such that $\frac{x}{(-\beta)^{k}}, \frac{y}{(-\beta)^{k}}, \frac{x+y}{(-\beta)^{k}} \in\left(\ell_{\beta}, \ell_{\beta}+1\right)$. By Lemma 11, we have

$$
T_{-\beta}^{j}\left(\frac{x}{(-\beta)^{k}}\right)+T_{-\beta}^{j}\left(\frac{y}{(-\beta)^{k}}\right)-T_{-\beta}^{j}\left(\frac{x+y}{(-\beta)^{k}}\right) \in \Phi(V)
$$

for all $j \geq 0$, thus $T_{-\beta}^{k}\left(\frac{x+y}{(-\beta)^{k}}\right) \in-\Phi(V)$. Therefore, we have $T_{-\beta}^{k}\left(\frac{x+y}{(-\beta)^{k}}\right)=\phi(\mathbf{z})=$ $-\Phi(-\mathbf{z}, h)$ for some $\mathbf{z}=\left(z_{0}, z_{1}\right) \in \mathbb{Z}^{2}$ and $h \in\{0,1\}$ with $(-\mathbf{z}, h) \in V$.

If $(\mathbf{z}, 0) \in V$, then the proof of Proposition 14 shows that $\tau_{\mathbf{r}, \alpha}^{\mu}(\mathbf{z})=\mathbf{0}$, thus $\operatorname{fr}(x+y) \leq \mu$. Assume now that $(\mathbf{z}, 0) \notin V$. Then

$$
-\mathbf{z} \in D_{m+1} \cup\{(-m-1,-j): 1 \leq j \leq m\} \cup\{(-j, m-j+1): 1 \leq j \leq m\} .
$$

We can exclude $-\mathbf{z}=(-j, m-j+1), 1 \leq j \leq m$, because this would imply $h=0$ and

$$
-\Phi(-\mathbf{z}, h)=1-\frac{j m}{\beta^{2}}-\frac{(m+1) m}{\beta^{3}} \geq \frac{m}{\beta}-\frac{m^{2}}{\beta^{3}}>\frac{1}{\beta+1} .
$$

This means that $\mathbf{z} \in\left(A_{m+1} \cup B_{m+1}\right) \backslash\{(-1, m+1),(m+1, m+1)\}$. With the notation of Lemma 13, we have

$$
A_{m+1} \backslash\{(-1, m+1)\} \rightarrow B_{m+1} \backslash\{(m+1, m+1)\} \rightarrow C_{m},
$$

where we have used Lemma 12 and that $\left\lfloor r_{0}(m+1)+r_{1} j+\alpha\right\rfloor=m-j$ for $1 \leq j \leq m$, as

$$
-\frac{\beta}{\beta+1}<\frac{m}{\beta}-\frac{m^{2}}{\beta^{2}}+\frac{m-m^{2}}{\beta^{3}} \leq \frac{m}{\beta}-\frac{m^{2}}{\beta^{2}}+\frac{(j-m) m}{\beta^{3}} \leq \frac{m}{\beta}-\frac{m^{2}}{\beta^{2}}<\frac{1}{\beta+1} .
$$

Hence, the points in $A_{m+1} \backslash\{(-1, m+1)\}$ and $B_{m+1} \backslash\{(m+1, m+1)\}$ are mapped to $(0,0)$ in the same number of steps as those in $A_{m}$ and $B_{m}$ respectively, thus $\operatorname{fr}(x+y) \leq \mu$. 
Now, Theorem 9 is an immediate consequence of Propositions 14 and 15.

Remark 16. It is also possible to determine the exact value of $\max \left\{\operatorname{fr}(x+y): x, y \in \mathbb{Z}_{-\beta}\right\}$ in the same fashion as in the proof of Proposition 14; we have

$$
\max \left\{\operatorname{fr}(x+y): x, y \in \mathbb{Z}_{-\beta}\right\}=3 m+ \begin{cases}1 & \text { if } m=2 \\ 2 & \text { if } m \geq 4 \text { is even } \\ 3 & \text { if } m=1 \\ 4 & \text { if } m \geq 3 \text { is odd. }\end{cases}
$$

\section{References}

[1] S. Akiyama, Cubic Pisot units with finite beta expansions, Algebraic number theory and Diophantine analysis (Graz, 1998), 11-26, de Gruyter, Berlin, 2000.

[2] S. Akiyama, T. Borbély, H. Brunotte, A. Pethő, J. Thuswaldner, Generalized radix representations and dynamical systems. I., Acta Math. Hungar. 108 (2005), no. 3, $207-238$.

[3] S. Akiyama, H. Brunotte, A. Pethő, J. Thuswaldner, Generalized radix representations and dynamical systems. II., Acta Arith. 121 (2006), no. 1, 21-61.

[4] S. Akiyama, K. Scheicher, Symmetric shift radix systems and finite expansions, Math. Pannon. 18 (2007), no. 1, 101-124.

[5] P. Ambrož, Ch. Frougny, Z. Masáková, E. Pelantová, Arithmetics on number systems with irrational bases, Bull. Soc. Math. Belg. 10 (2003), 641-659.

[6] J. Bernat, Computation of $L_{\oplus}$ for several cubic Pisot numbers, Discrete Math. Theor. Comput. Sci. 9 (2007), no. 2, 175-193.

[7] H. Brunotte, Symmetric CNS trinomials, INTEGERS 9 (2009), A19, 201-214.

[8] D. Dombek, Z. Masáková, T. Vávra, Confluent Parry numbers, their spectra, and integers in positive- and negative-base number systems, J. Théor. Nombres Bordeaux 27 (2015), 745-768.

[9] K. Dajani, Ch. Kalle, Transformations generating negative $\beta$-expansions, INTEGERS 11B (2011), A5.

[10] S. Dammak, M. Hbaib, Number systems with negative bases, Acta Math. Hungar. 142 (2014), no. 2, 475-483.

[11] Ch. Frougny, Confluent Linear Numeration Systems, Theor. Comput. Sci. 106 (1992), no. 2, 183-219. 
[12] Ch. Frougny, A. C. Lai, Negative bases and automata, Discrete Math. Theor. Comput. Sci. 13 (2011), no. 1, 75-93.

[13] Ch. Frougny, B. Solomyak, Finite beta-expansions, Ergodic Theory Dynam. Systems 12 (1992), no. 4, 713-723.

[14] P. J. Grabner, A. Pethő, R. F. Tichy, G. J. Wöginger, Associativity of recurrence multiplication, Appl. Math. Letters 7 (1994), no. 4, 85-90.

[15] T. Hejda, E. Pelantová, Spectral Properties of Cubic Complex Pisot Units, Math. Comp. 85 (2016), 401-421.

[16] M. Hollander, Linear numeration systems, finite beta expansions, and discrete spectrum of substitution dynamical systems, Ph.D. Thesis, University of Washington, 1996.

[17] S. Ito and T. Sadahiro, Beta-expansions with negative bases, INTEGERS 9 (2009), 239-259.

[18] P. Kirschenhofer and J. M. Thuswaldner, Shift radix systems - a survey, Numeration and substitution 2012, 1-59, RIMS Kôkyûroku Bessatsu, B46, Res. Inst. Math. Sci. (RIMS), Kyoto, 2014.

[19] Z. Masáková, E. Pelantová, Purely periodic expansions in systems with negative base, Acta Math. Hungar. 139 (2013), no. 3, 208-227.

[20] Z. Masáková, E. Pelantová, T. Vávra, Arithmetics in number systems with negative base, Theor. Comp. Sci. 412 (2011), no. 8-10, 835-845.

[21] A. Messaoudi, Tribonacci multiplication, Appl. Math. Lett. 15 (2002), 981-985.

[22] W. Parry, On the $\beta$-expansions of real numbers, Acta Math. Acad. Sci. Hungar. 11 (1960), 401-416.

[23] A. Rényi, Representations for real numbers and their ergodic properties, Acta Math. Acad. Sci. Hung. 8 (1957), 477-493.

[24] P. Surer, $\epsilon$-shift radix systems and radix representations with shifted digit sets, Publ. Math. Debrecen 74 (2009), no. 1-2, 19-43. 